\title{
Differential Impact of COVID-19 Risk Factors on Ethnicities in the United States
}

OPEN ACCESS

Edited by:

Jie Hu,

The Ohio State University,

United States

Reviewed by:

Samuel Manda,

South African Medical Research

Council, South Africa

Shivani Sharma,

University of Hertfordshire,

United Kingdom

*Correspondence:

Sumona Mondal

smonda/@clarkson.edu

Shantanu Sur

ssur@clarkson.edu

tThese authors have contributed equally to this work

Specialty section:

This article was submitted to Life-Course Epidemiology and Social Inequalities in Health,

a section of the journal

Frontiers in Public Health

Received: 17 July 2021 Accepted: 10 November 2021 Published: 06 December 2021

Citation:

Athavale P, Kumar V, Clark J Mondal S and Sur S (2021) Differential Impact of COVID-19 Risk Factors on

Ethnicities in the United States.

Front. Public Health 9:743003.

doi: 10.3389/fpubh.2021.743003

\author{
Prashant Athavale ${ }^{1 \dagger}$, Vijay Kumar ${ }^{1 \dagger}$, Jeremy Clark ${ }^{1}$, Sumona Mondal ${ }^{1 *}$ and Shantanu Sur ${ }^{2 *}$ \\ ${ }^{1}$ Department of Mathematics, Clarkson University, Potsdam, NY, United States, ${ }^{2}$ Department of Biology, Clarkson University, \\ Potsdam, NY, United States
}

The coronavirus disease (COVID-19) has revealed existing health inequalities in racial and ethnic minority groups in the US. This work investigates and quantifies the non-uniform effects of geographical location and other known risk factors on various ethnic groups during the COVID-19 pandemic at a national level. To quantify the geographical impact on various ethnic groups, we grouped all the states of the US. into four different regions (Northeast, Midwest, South, and West) and considered Non-Hispanic White (NHW), NonHispanic Black (NHB), Hispanic, Non-Hispanic Asian (NHA) as ethnic groups of our interest. Our analysis showed that infection and mortality among NHB and Hispanics are considerably higher than NHW. In particular, the COVID-19 infection rate in the Hispanic community was significantly higher than their population share, a phenomenon we observed across all regions in the US but is most prominent in the West. To gauge the differential impact of comorbidities on different ethnicities, we performed cross-sectional regression analyses of statewide data for COVID-19 infection and mortality for each ethnic group using advanced age, poverty, obesity, hypertension, cardiovascular disease, and diabetes as risk factors. After removing the risk factors causing multicollinearity, poverty emerged as one of the independent risk factors in explaining mortality rates in $\mathrm{NHW}, \mathrm{NHB}$, and Hispanic communities. Moreover, for $\mathrm{NHW}$ and $\mathrm{NHB}$ groups, we found that obesity encapsulated the effect of several other comorbidities such as advanced age, hypertension, and cardiovascular disease. At the same time, advanced age was the most robust predictor of mortality in the Hispanic group. Our study quantifies the unique impact of various risk factors on different ethnic groups, explaining the ethnicity-specific differences observed in the COVID-19 pandemic. The findings could provide insight into focused public health strategies and interventions.

Keywords: COVID-19, infection, mortality, ethnicity, Hispanic, risk factors, diabetes

\section{INTRODUCTION}

Numerous researchers have found various comorbidities and other risk factors affecting the spread and prognosis of coronavirus disease (COVID-19). Recent work by many researchers has also demonstrated that the COVID-19 pandemic has affected marginalized ethnicities more severely. We thus hypothesize that the risk factors for COVID-19 must have affected different ethnic groups in a distinctive manner. In this paper, we aim to quantify the differential effect of risk factors on different ethnicities. 


\subsection{COVID-19 and Ethnicity}

The public health crisis created by the COVID-19 has uncovered the historical inequalities (1-4) between ethnic groups in certain countries, in particular in the UK and US, which are countries with ethnically diverse populations. These observations and consistent fatal outcomes in the minority ethnic groups $(5,6)$ have led to speculations about why patients from these groups are susceptible to infections, followed by severe complications. These trends could be due to different rates of COVID19 infections, underlying health conditions, living conditions including housing density, having jobs as essential workers, access to health care, quality of care, and a mixture of multiple factors among these groups. The United States national data (7) from states and municipalities reports disproportionate COVID-19 infections, hospitalizations, and deaths among minority ethnic groups. Dobin and Dobin (8) showed that the infection rate is 4 -fold for the Black and Hispanic population in selected counties in New York state. Moore et al. (9) observe a disproportionate number of COVID-19 cases among underrepresented racial/ethnic groups in the United States. Adhikari et al. (10) show that the racial and ethnic disparities in COVID-19 infections and deaths existed beyond those explained by income inequality.

\subsection{Effects of Geographical Location on COVID-19}

The impact of COVID-19 varies widely across countries and even within a country or a region. For example, Sun et al. (11) showed a negative correlation between the number provincial COVID19 cases and latitude, as well as altitude. Breen and Ermisch (12) use spatial autoregressive regression to show that the relation of COVID-19 mortality to social composition of geographical areas in England is distinct than that of non-COVID mortality. A number of factors including societal awareness and culture, public health measures, healthcare infrastructure, and more recently vaccination coverage are known underlie the variation for COVID-19 infection rates and adverse health outcome (13).

Although multiple studies have confirmed that black and Hispanic populations in the US are more vulnerable to COVID19 , to our knowledge, no data is available if they are equally susceptible across geographical locations. Stephens-Davidowitz (14) uses the search data from Google to show that there exists a wide variation in racism in the US within the 50 states. Thus, we surmise that the impact of the COVID-19 on various ethnicities may not be uniform in all regions of the US. Hence, we are interested in understanding if a geographical location plays a part in the variation of COVID-19 impact on minorities.

\subsection{Comorbidities for COVID-19}

Emerging evidence highlights that comorbid conditions such as obesity, cardiovascular disease (CVD), and type 2 diabetes are directly linked to the severity of the COVID-19 disease (1517). A meta-analysis including 76,993 patients with COVID-19 showed diabetes, CVD, smoking, malignancy, chronic kidney disease, hypertension, chronic obstructive pulmonary disease (COPD) are associated with poor prognosis (18). This conclusion was further supported by Richardson et al. (19), and Sun et al. (20). Using logistic regression (21) show that obesity was a risk factor for the severity of the COVID-19 disease. Furthermore, in a retrospective cohort study, Busetto et al. (22) conclude that despite their young age, overweight patients were more likely to need assisted ventilation and access to intensive care units than patients with normal weight. The connection between obesity and pulmonary function is wellestablished, e.g., Sharp et al. (23) observe that obese patients have significantly decreased total respiratory compliance. Moreover, Li et al. (24) find that reduction in functional residual capacity and diffusion impairment are the most common abnormalities in obese patients. Yan et al. (18) show that diabetic patients experienced more mortality than non-diabetic patients. Finally, just as in the case of the SARS epidemic (25), COVID-19 has disproportionately affected the older population (26). In fact, in the US, $92 \%$ of the COVID-19 recorded deaths till June, 2020 are in the age group 55 years and above (27). In summary, the main comorbidities for COVID-19 include obesity, diabetes, advanced age, hypertension, and cardiovascular disease.

However, these risk factors affect different ethnicities differently. For example, Paeratakul et al. (28) find that among obese individuals, the prevalence of hypertension was higher in NHB subjects than other groups. Sturm and Hattori (29) observe that the prevalence of obesity is about double among NHB than among Hispanics or NHW. Kuzawa and Sweet (30) note that NHB suffer from a disproportionate burden of CVD relative to NHW. Thus, we are motivated to understand whether or not these comorbidities affect different ethnicities differently.

\subsection{Impact of Poverty on COVID-19 Prognosis}

Patel et al. (31) note that economically disadvantaged people are vulnerable to COVID-19 due to a combination of factors. A time-series analysis conducted by Elgar et al. (32) reveals that income inequality is associated with a higher number of deaths due to COVID-19 in 84 countries. In particular, in the US, the states with higher income inequality experienced a higher rate of infection as well as the number of COVID-19 related deaths (33). This pattern could be because the comorbidities associated with COVID-19 are linked to poverty.

A longitudinal study involving 600,662 adults from Taiwan's National Healthcare Insurance database indicates that diabetes incidence is associated with poverty (34). This finding is particularly notable since the subjects from this study had access to universal healthcare. However, the subjects were from a ethnically homogeneous population. Thus, we are motivated to investigate the differential role of poverty among various races.

\subsection{Objectives}

For this study, we choose Non-Hispanic white (NHW), NonHispanic Black (NHB), Hispanic, and Non-Hispanic Asian (NHA) as four ethnic groups. The risk factors we choose to focus on in this work are advanced age, obesity, cardiovascular disease, diabetes, hypertension, and poverty. We aim to investigate the following in this study: 
1. Does a geographical location have different impact on COVID-19 infection and mortality rates for different ethnicities?

2. Do various COVID-19 risk factors have a different effect on different ethnicities?

To this end, we collected COVID-19 related infections and mortality data from various publicly available sources, as described in section 2.1. To investigate the geographical variation in the impact of COVID-19, we seek to quantify the difference between the distributions of infection rates, mortality rates, and populations of various ethnic groups in four geographical regions of the US (35). We describe our approach for this analysis in section 2.4.1. Finally, in section 2.4 .3 we construct robust linear models with infection and mortality rates of various ethnicities as response variables.

\section{MATERIALS AND METHODS}

\subsection{Selection of Variables and Data Sources}

Given the discussion in the sections 1.3, 1.1, and 1.4, we focus on the following factors in this work: obesity, diabetes, poverty, advanced age, hypertension, and cardiovascular diseases. We included the following ethnic groups in our work: NHW, NHB, Hispanic, and NHA. We excluded the groups American Indian/Alaska Native, and Native Hawaiian/Other Pacific Islander from this work due to lack of reliable and consistent data sets in the US, cf. (36-38). We used the American Community Survey (39) census database to collect the following information for each state: NHW, NHB, Hispanic, and NHA population and their respective percentage contribution to the population of each state. To investigate the geographical effect on COVID-19 prognosis, we used the classification of the US into the following categories: Northeast, South, Midwest, and West (35). COVID-19 infection and death counts between January 21, 2020, to September 30, 2020 from KFF Covid-19 data (40) were obtained. The time window roughly corresponds to the first pandemic wave experienced in the US. For each ethnicity E, and for a specific state $\boldsymbol{S}$ the variable Relative Infection $\%$ is defined as follow:

$$
\begin{aligned}
& \text { Relative Infection } \% \\
& :=\frac{\text { COVID-19 positive subjects of ethnicity } \mathbf{E} \text { in state } \mathbf{S}}{\text { Total number of COVID-19 positive subjects in state } \mathbf{S}} \times 100 \text {. }
\end{aligned}
$$

We note that the choice of "relative" infection percentage as a response variable is deliberate. This choice allows us to directly compare this number with the population share of that ethnicity in the region. Similarly, for each ethnicity $\mathbf{E}$, and for a specific state $\mathbf{S}$ we define the variable Relative Mortality as follow:

$$
\begin{aligned}
& \text { Relative Mortality } \% \\
& :=\frac{\text { COVID-19 related deaths for ethnicity } \mathbf{E} \text { in state } \mathbf{S}}{\text { Total number of COVID-19 deaths in state } \mathbf{S}} \times 100 \text {. }
\end{aligned}
$$

In our work, Relative Infections $\%$ and Relative Mortality $\%$ were considered as the response variables. For brevity, we write infection rate instead of Relative Infections $\%$, and so on. The use of relative percentages allows a direct comparison with the population percentages of that ethnicity. For example, in a state with a 5\% NHB population, relative mortality of $15 \%$ in the NHB community indicates disproportionately large mortality compared to the NHB population. The use of "relative" percentage is independent of the population of the state itself. The use of this measure also allows us to compare the impact on a certain ethnic group in two states with similar proportion of the minority population. As a concrete example, when we consider the states of California and Texas, both have a similar percentage of the Hispanic population, 39.5 and $40 \%$, respectively. However, the relative mortality percentages for the Hispanic group in California and Texas are 48.3 and $56.1 \%$, respectively. We collected the data on the percentage of people with age 60 or more in each state and ethnicity is obtained from the CDC dataset (41). Race and state-wise data were obtained from adults who reported being told by a health professional that they have diabetes (excluding prediabetes and gestational diabetes) using the America's Health Rankings (42). We used the body mass index (BMI) as a measure of obesity following (43) and define obesity as a condition of having a BMI of 30.0 or higher. The dataset (44) were used to obtain the obesity data from each state and for the races NHW, NHB, Hispanic, NHA. We acquired the percentage of adults whom a health care professional informed that they had a coronary heart disease, or myocardial infarction, or a stroke from AHR CVD data (45). This was gathered for each state and ethnicity of interest. We obtained the race and state-wise data on adults who reported being informed by a health professional that they have high blood pressure from AHR HBP data (46). The US Census Bureau defines the "poverty threshold" for a family with two adults and one child as $\$ 20,578$ in 2019 . We extracted the data from KFF Poverty data (47) on poverty defined by the "poverty threshold." We obtained this data for each state and ethnicity of interest.

For each state, and each of the four ethnicity of interest (NHW, NHB, Hispanic, and NHA) we defined the variables: Age $60+$, BMI30+ (a measure of obesity), CVD, Diabetes, HBP, Poverty. For a state $\mathbf{S}$, and an ethnicity $\mathbf{E}$ we defined the relative percentage of people with age 60 or over Age $60+$ as follows:

\footnotetext{
Relative Age60+ \%

$:=\frac{\text { Number of people of ethnicity } \mathbf{E} \text { with age over } 60 \text { in state } \mathbf{S}}{\text { Total number of people with age over } 60 \text { in state } \mathbf{S}} \times 100$.
}

We use the variable name Age $60+$ instead of Relative Age $60+\div$ for conciseness, and so on. We define the relative percentage variables Obesity, CVD, Diabetes, HBP, and Poverty in a similar manner. 


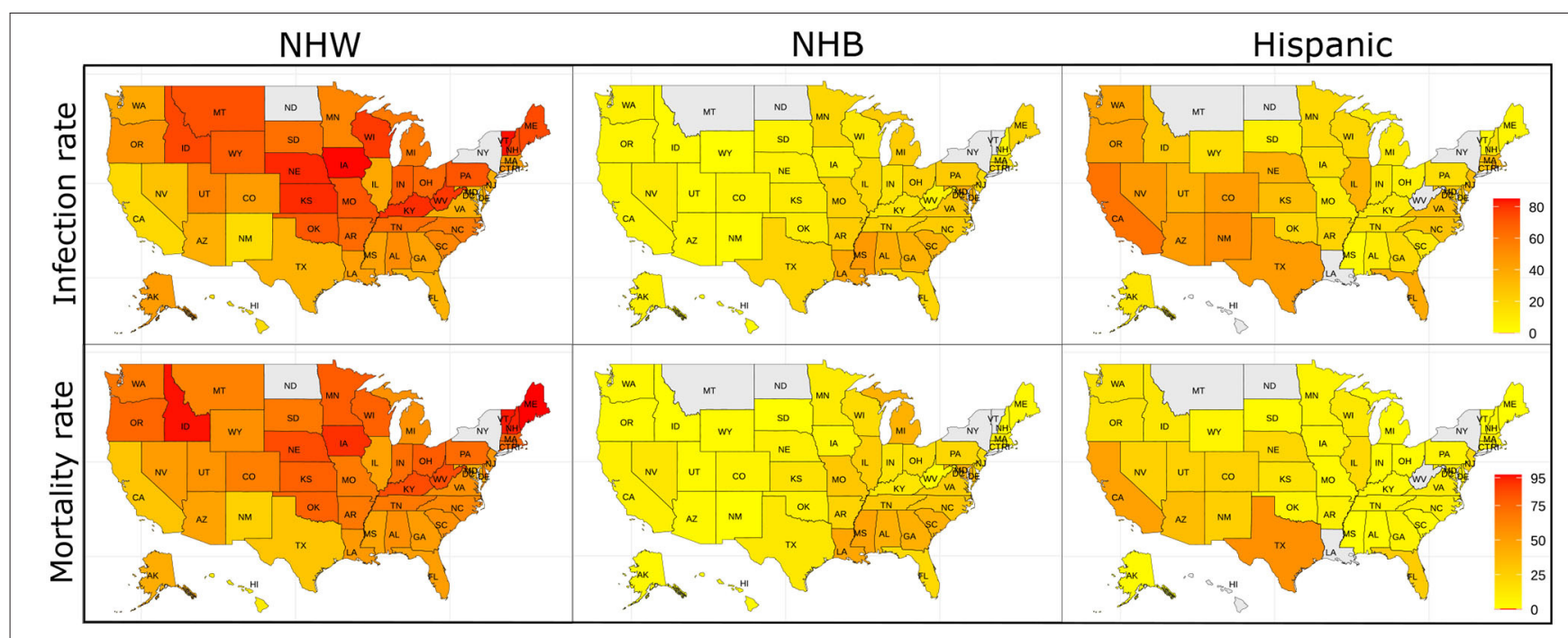

FIGURE 1 | The relative infection \% and relative mortality \% amongst the NHW, NHB, and Hispanic groups across the US. The states with no color indicate that the ethnicity-wise data on relative infection $\%$ and relative mortality $\%$ were not available in those states.

\subsection{A Note on Unavailability of Data From Some States}

We encountered a few irregularities during our data collection process in the format the data was made available by various states (36-38). For example, New York state does not provide ethnicity-wise COVID-19 infection and mortality data. Similarly, infection data for NHW communities only are available for North Dakota. Data availability of specific variables for different ethnicities also varied across states. Data for all variables for the NHW group could be obtained from 48 states whereas for the NHB and Hispanic communities such data were available from only 38 and 33 states, respectively. In our analysis, we included the states for which data for all the variables are available. Hatcher et al. (37) find that only 23 state in the US have complete data for American Indian and Alaska Native Persons.

\subsection{Description of Data}

The data for this study are state-level demographics based on four ethnic groups. We depict the relative infection $\%$ and relative mortality \% for NHW, NHB, and Hispanic group in the map in Figure 1. As described in section 2.2 some states do not make the ethnicity-wise data public. The states with no color in the Figure 1 indicate that the ethnicity-wise infection and mortality data was not available in those states. We calculated state-wise descriptive statistics for the relative infection and mortality percentages and population comparing each ethnic group. We performed a descriptive analysis to explore the region-specific, state-wise characteristics of for the relative infection $\%$ and mortality $\%$ and population by calculating their medians, first and third quartiles, and presented in Figure 2.

\subsection{Analytical Approach \\ 2.4.1. Quantifying the Regional Variability of COVID-19 on Various Races}

The infection and mortality rates for various ethnic groups are disproportionate to their share of the population in the US $(7,8)$. We aim to understand this phenomenon and its severity across various regions in the US. To this effect, we employed the Kruskal-Wallis (KW) test (48), a non-parametric equivalent of the one-way analysis of variance. Since the test does not identify the groups that differ in their distributions, we followed it with Dunn's multiple comparisons test (49) for cases for which the KW test yielded statistically significant results. We used the combination of KW test, and Dunn's comparison test for the groups NHW, NHB, Hispanic, and NHA separately for all four regions of the US, as well as the whole country.

\subsubsection{Correlation Analyses}

In order to quantify the association between the impact of COVID-19 on the ethnic groups and the risk factors across the country, we consider each state, for which the data are available, as a data point. We computed the pairwise Pearson's correlation coefficients between various risk factors for the racial groups NHW, NHB, and Hispanic, along with their 2-tailed statistical significance values. We summarized the comparisons between the variables in correlation matrices.

\subsubsection{Constructing Robust Linear Models With Infection and Mortality Rates as Response Variables}

In order to elucidate the role of the explanatory variables on a specific aspect of the COVID-19 burden linear models are employed. For these linear models, we considered each state as a data point. From the Figure 2, we observe that the rate of infection and mortality in the NHA are consistently lower when compared to their population. Thus, we consider building 


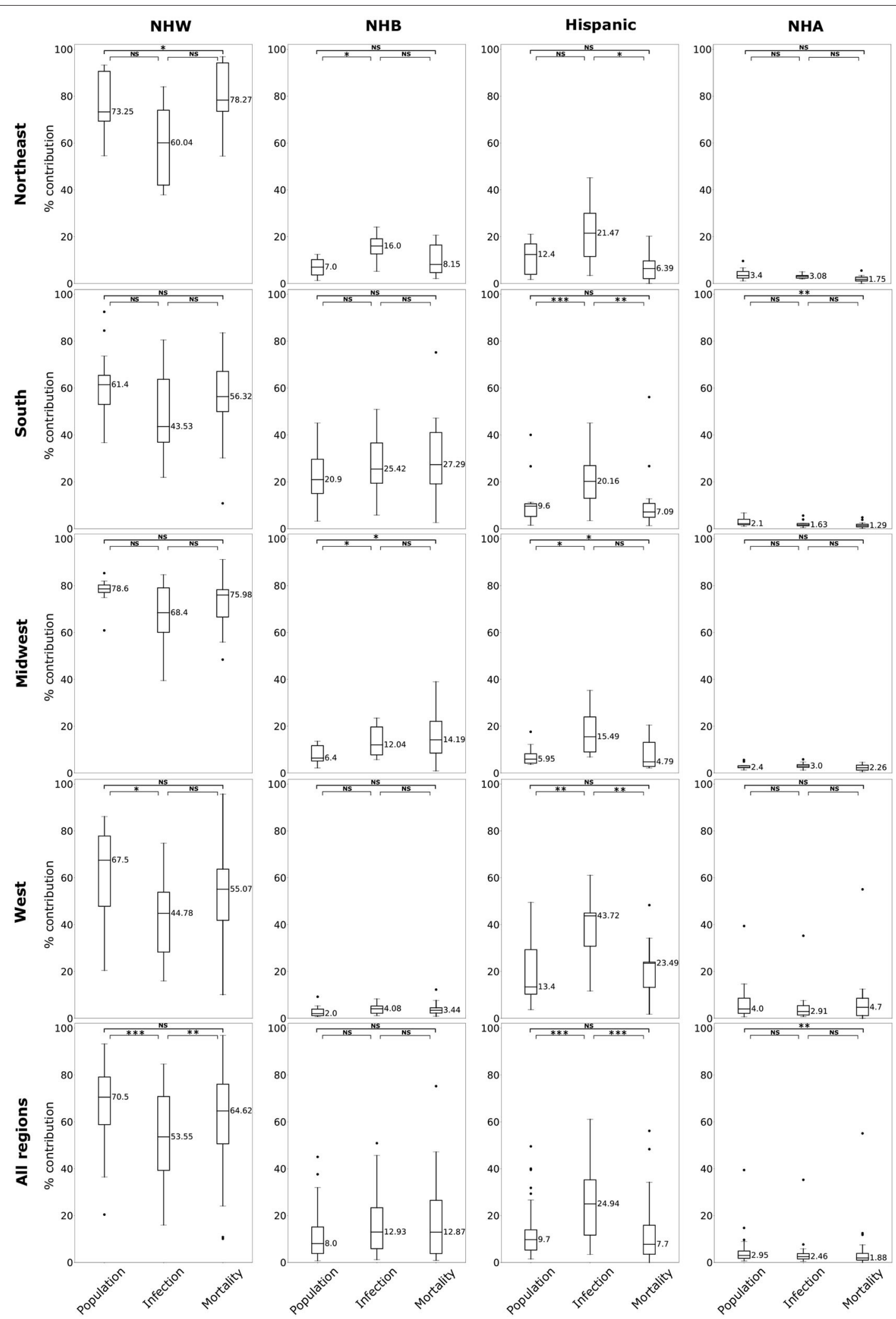

FIGURE 2 | Box plots of population, relative infection \%, and relative mortality \% in each of four US regions, and combining all regions for NHW, NHB, Hispanic and

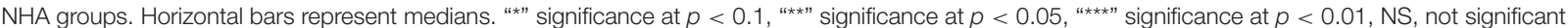
(Kruskal-Wallis tests followed by Dunn's tests). 
linear models for NHW, NHB, and Hispanic groups only to elucidate the contributions of risk factors considered in this study. However, infection and mortality rates, the response variables for our model, showed skewness in their distributions. Since logarithmic (log) transformation of data is one of the most commonly used techniques to conform to normality (50), we implemented it on infection and mortality rates. The log transformation was effective in correcting the skewness and introduce normality (Supplementary Figures S1, S2). As log transformation of infection and mortality rates improved their normality behavior, we used log transformed form of these variables exclusively for model construction. Thus, when we refer to infection rate and mortality rate in the context of linear models they denote log transformed infection rate and mortality rate, respectively. For NHW, NHB, and Hispanic groups, we built our preliminary linear models with infection rate, and mortality rate as our response variables and the risk factors defined in section 1.3, i.e., advanced age Age $60+$, BMI $30+$ (a measure of obesity), CVD, Diabetes, HBP, Poverty as the explanatory variables.

However, conditions of advanced age, cardiovascular disease, diabetes, obesity, and hypertension are interrelated. This interrelation can also be observed from the correlation Tables 35. Multicollinearity among the explanatory variables can lead to unstable and unreliable estimates of regression coefficients (51). We used the variance inflation factor (VIF) to assess the multicollinearity between the explanatory variables (52). Following Kutner et al. (53, p. 409) an upper cut-off value of VIF for explanatory variables is set as 10 to minimize the contribution of multicollinearity in our model. Starting from the preliminary model for ethnicity of interest, we propose the procedure outlined below to construct our final model:

1. Compute the VIF for each explanatory variable in the model. If all the VIFs are less than 10, we declare this to be the final linear model.

2. If an explanatory variable has a VIF of more than 10 , we remove the explanatory variable with the largest VIF. If there are more than one explanatory variables with VIF within 5\% of the maximum VIF, we remove the variable that leads to a model with the highest adjusted $R^{2}$.

3. We construct the linear model with the remaining explanatory variables. After the removal of a variable, it is possible to include more data points in our model. For example, after removing the variable $\mathrm{Di}$ abetes, we could include states for which data on diabetes was not available.

4. Go to Step 2.4.3.

After constructing the linear models, we checked the normality of the residuals of the regression models with Lilliefors normality test (54).

\subsection{Geographically Weighted Regression}

Linear regression yields stationary and global regression coefficients. However, it is conceivable that these coefficients might have local variability. To find the geographical variability in the coefficients, we employed the geographically weighted regression (GWR) (55). Rather than producing global regression results, GWR yields "local" regression coefficients in terms of geographically varying functions. For our analysis, we used the infection rate and mortality rates as response variables and the variables obtained from section 2.4.3 as the explanatory variables.

\subsection{Coding Language and Libraries Used}

For our coding, we used $\mathrm{R}$ language (version 4.0.0), along with the following libraries in our coding: readxl, dplyr, tidyr, FSA, ggplot2, car, qqplotr, nortest, pwr, spgwr, sp, sf, rgdal, rgeos, tmap, tmaptools.

\section{RESULTS}

\subsection{Regional Variation of COVID-19 Impact on Various Ethnicities}

The boxplots in Figure 2 summarize the relative impact of COVID-19 on various ethnicities across the four regions of the US and all regions as an aggregate. In Figure 2 we present various descriptive statistics of the population, infection, and mortality rates for the NHW, NHB, Hispanic, and the NHA groups across various regions. As noted in the section 2.2, not all the states are included in the analyses. Thus, the statistics shown in this plot do not correspond closely to those of the whole country. We describe the Kruskal-Wallis test results in Table $\mathbf{1}$. We see in Table 1 that the KW test for NHW is statistically significant in the Northeast and the West with $p<0.1$. For the NHB group, the KW test is significant in the Northeast and the Midwest with $p<0.1$. The KW test was statistically significant for the Hispanic group in "all four regions," with $p<0.1$ in the Northeast; with $p<0.05$ in Midwest and the West; and $p<0.01$ in the South. The NHA data yielded significant results with the KW test only in the South with $p<0.05$.

When we considered all four regions in the US together, the KW test was statistically significant for all ethnicities with $p<0.01$ for NHW and Hispanic communities. The KW test was significant for the NHB and NHA when all regions were combined with $p<0.1$.

We followed the significant KW tests with Dunn's multiple comparison test to identify factors differing in their distributions. We depict the results from the Dunn's test in Table 2. In particular, we obtained statistically significant results (with $p<0.05$ ) in the South, Midwest, and West for the Hispanic population between the pairs 'infection \& mortality rates' and "infection rate and population share."

\subsection{Results of the Correlation Analyses}

The KW test provides evidence of geographical impact on various ethnicities. In this section we provide the results of correlation analysis between other risk factors. In Table 3 we see the Pearson correlations between the variables along with the 2-tailed significance values for the NHW group. The same statistics are provided in Tables 4, 5 for the NHB and Hispanic communities respectively. All the variables are strongly $(p<$ 0.01 ) and positively correlated with every other variable for all ethnicities, with poverty being the sole exception. To be precise, for the NHW group, poverty is positively correlated 


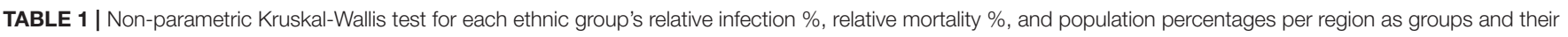
significance levels.

\begin{tabular}{|c|c|c|c|c|c|c|c|c|}
\hline & \multicolumn{2}{|c|}{ NHW } & \multicolumn{2}{|c|}{ NHB } & \multicolumn{2}{|c|}{ Hispanic } & \multicolumn{2}{|c|}{ NHA } \\
\hline & Statistic & $p$-value & Statistic & $p$-value & Statistic & $p$-value & Statistic & $p$-value \\
\hline Northeast & $5.38^{\star}$ & 0.068 & $5.43^{\star}$ & 0.066 & $5.21^{*}$ & 0.074 & 3.81 & 0.148 \\
\hline South & 4.37 & 0.112 & 2.95 & 0.228 & $10.96^{\star \star \star}$ & 0.004 & $7.77^{\star \star}$ & 0.020 \\
\hline Midwest & 3.88 & 0.137 & $5.83^{\star}$ & 0.054 & $8.66^{\star \star}$ & 0.013 & 2.40 & 0.302 \\
\hline West & $4.88^{\star}$ & 0.087 & 2.02 & 0.364 & $8.83^{\star \star}$ & 0.012 & 0.60 & 0.741 \\
\hline All regions & $13.03^{\star \star \star}$ & 0.002 & $4.83^{\star}$ & 0.089 & $26.18^{\star \star \star}$ & $<0.01$ & $5.38^{\star}$ & 0.067 \\
\hline
\end{tabular}

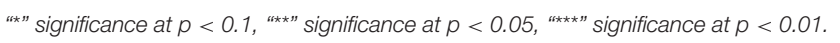

TABLE 2 | Post-hoc analysis using Dunn Test and their significance levels.

\begin{tabular}{|c|c|c|c|c|c|c|}
\hline \multirow[b]{2}{*}{ Ethnic group-region } & \multicolumn{2}{|c|}{ Infection\%-Mortality\% } & \multicolumn{2}{|c|}{ Infection\%-Population } & \multicolumn{2}{|c|}{ Mortality\%-Population } \\
\hline & Statistic & $p$-value & Statistic & $p$-value & Statistic & $p$-value \\
\hline NHW-Northeast & $-2.30^{\star}$ & 0.064 & -1.41 & 0.314 & 0.88 & 0.377 \\
\hline NHW-South & - & - & - & - & - & - \\
\hline NHW-Midwest & - & - & - & - & - & - \\
\hline NHW-West & -1.09 & 0.275 & $-2.21^{\star}$ & 0.081 & -1.12 & 0.527 \\
\hline NHW-All & $-2.29^{\star \star}$ & 0.043 & $-3.55^{\star \star \star}$ & 0.001 & -1.26 & 0.207 \\
\hline NHB-Northeast & 1.29 & 0.392 & $2.32^{\star}$ & 0.060 & 1.03 & 0.301 \\
\hline NHB-South & - & - & - & - & - & - \\
\hline NHB-Midwest & -0.19 & 0.842 & $1.09^{\star}$ & 0.094 & $2.18^{\star}$ & 0.087 \\
\hline NHB-West & - & - & - & - & - & - \\
\hline NHB-All & 0.37 & 0.711 & 2.06 & 0.117 & 1.69 & 0.181 \\
\hline Hispanic-Northeast & $2.27^{\star}$ & 0.068 & 1.31 & 0.375 & -1.02 & 0.305 \\
\hline Hispanic-South & $2.88^{\star \star}$ & 0.011 & $2.87^{\star \star \star}$ & 0.008 & -0.10 & 0.917 \\
\hline Hispanic-Midwest & $2.67^{\star \star}$ & 0.022 & $2.42^{\star \star}$ & 0.031 & -0.31 & 0.753 \\
\hline Hispanic-West & $2.33^{\star \star}$ & 0.039 & $2.78^{\star \star}$ & 0.015 & 0.36 & 0.718 \\
\hline Hispanic-All & $4.60^{\star \star \star}$ & $<0.01$ & $4.28^{\star \star \star}$ & $<0.01$ & -0.47 & 0.638 \\
\hline NHA-Northeast & - & - & - & - & - & - \\
\hline NHA-South & 1.22 & 0.221 & -1.55 & 0.239 & $-2.78^{\star \star}$ & 0.016 \\
\hline NHA-Midwest & - & - & - & - & - & - \\
\hline NHA-West & - & - & - & - & - & - \\
\hline NHA-All & 1.39 & 0.325 & -1.30 & 0.190 & $-2.7^{\star \star}$ & 0.020 \\
\hline
\end{tabular}

“*” significance at $p<0.1$, “**” significance at $p<0.05$, "“***” significance at $p<0.01$.

with the infection rate $(r=0.35, p<0.05)$, and diabetes $(r=0.29, p<0.05)$. Poverty is either uncorrelated or weakly correlated with other variables in this study for all ethnicities.

\subsection{Results of the Linear Models With Infection and Mortality Rates as Response Variables for Each Ethnic Group}

In Table 6 we depict the linear models with infection rate and mortality rate as response variables for NHW. The first column shows preliminary models along with the VIFs for each explanatory variable. The second column depicts the final model obtained via the maximum VIF elimination algorithm described in section 2.4.3. The preliminary model with infection rates in the NHW community as the response variable accounts for $83 \%\left[R^{2}=0.83, R_{a d j}^{2}=0.80, F_{(6,41)}=32.87, p<0.01\right]$ of the variability in the infection rates for NHW population. The final model for the NHW infection rates consists of obesity, diabetes, and poverty as the only explanatory variables. This model accounts for $82 \%\left[R^{2}=0.82, R_{a d j}^{2}=0.80, F_{(3,44)}=\right.$ $65.06, p<0.01$ ] of the variability in the NHW infection rates. The final NHW infection model and preliminary model both use 48 states.

The preliminary model with NHW mortality rates as the response variable accounts for $88 \%\left[R^{2}=0.88, R_{a d j}^{2}=\right.$ $\left.0.87, F_{(6,411)}=51.95, p<0.01\right]$ of the variability in the NHW mortality rates. The final model for the NHW mortality also consists of obesity, diabetes, and poverty as the only explanatory 
TABLE 3 | Pearson correlations for NHW ethnic group between variables used in the study and their significance levels.

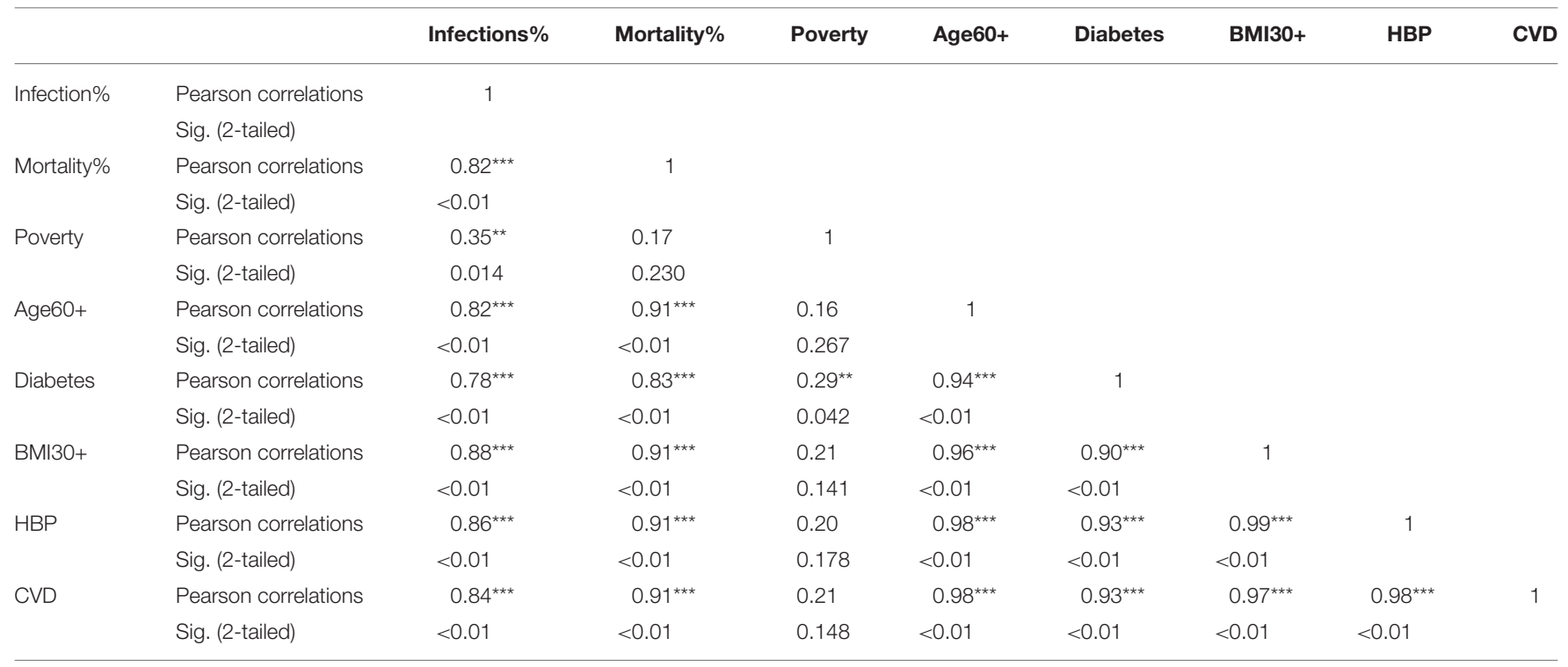

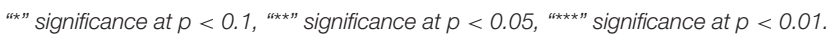

TABLE 4 | Pearson correlations for NHB ethnic group between variables used in the study and their significance levels.

\begin{tabular}{|c|c|c|c|c|c|c|c|c|c|}
\hline & & Infection $\%$ & Mortality\% & Poverty & Age60+ & Diabetes & BMI30+ & HBP & CVD \\
\hline \multirow[t]{2}{*}{ Infection\% } & Pearson correlations & 1 & & & & & & & \\
\hline & Sig. (2-tailed) & & & & & & & & \\
\hline \multirow[t]{2}{*}{ Mortality\% } & Pearson correlations & $0.93^{\star \star \star}$ & 1 & & & & & & \\
\hline & Sig. (2-tailed) & $<0.01$ & & & & & & & \\
\hline \multirow[t]{2}{*}{ Poverty } & Pearson correlations & -0.03 & -0.07 & 1 & & & & & \\
\hline & Sig. (2-tailed) & 0.833 & 0.677 & & & & & & \\
\hline \multirow[t]{2}{*}{ Age60+ } & Pearson correlations & $0.90^{\star \star \star}$ & $0.95^{\star \star \star}$ & -0.10 & 1 & & & & \\
\hline & Sig. (2-tailed) & $<0.01$ & $<0.01$ & 0.550 & & & & & \\
\hline \multirow[t]{2}{*}{ Diabetes } & Pearson correlations & $0.92^{\star \star \star}$ & $0.95^{\star \star \star}$ & -0.084 & $0.99^{\star \star \star}$ & 1 & & & \\
\hline & Sig. (2-tailed) & $<0.01$ & $<0.01$ & 0.610 & $<0.01$ & & & & \\
\hline \multirow[t]{2}{*}{ BMI30+ } & Pearson correlations & $0.94^{\star \star \star}$ & $0.96^{\star \star \star}$ & -0.09 & $0.99^{\star \star \star}$ & $0.99^{\star \star \star}$ & 1 & & \\
\hline & Sig. (2-tailed) & $<0.01$ & $<0.01$ & 0.592 & $<0.01$ & $<0.01$ & & & \\
\hline \multirow[t]{2}{*}{ HBP } & Pearson correlations & $0.94^{\star \star \star}$ & $0.96^{\star \star \star}$ & -0.09 & $0.99^{\star \star \star}$ & $0.99^{\star \star \star}$ & $0.99^{\star \star \star}$ & 1 & \\
\hline & Sig. (2-tailed) & $<0.01$ & $<0.01$ & 0.592 & $<0.01$ & $<0.01$ & $<0.01$ & & \\
\hline \multirow[t]{2}{*}{ CVD } & Pearson correlations & $0.86^{\star \star \star}$ & $0.92^{\star \star \star}$ & 0.07 & $0.99^{\star \star \star}$ & $0.98^{\star \star \star}$ & $0.96^{\star \star \star}$ & $0.97^{\star \star \star}$ & 1 \\
\hline & Sig. (2-tailed) & $<0.01$ & $<0.01$ & 0.691 & $<0.01$ & $<0.01$ & $<0.01$ & $<0.01$ & \\
\hline
\end{tabular}

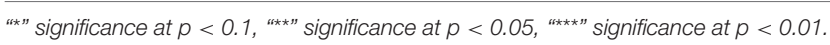

variables. This NHW mortality final model accounts for $7 \%$ $\left[R^{2}=0.78, R_{a d j}^{2}=0.77, F_{(3,44)}=53.48, p<0.01\right]$ of the variability in the NHW mortality.

In Table 7 we depict the linear models with infection rate and mortality rate as response variables for NHB. The preliminary model with infection rates in the NHB group as the response variable accounts for $81 \%\left[R^{2}=0.81, R_{a d j}^{2}=0.77, F_{(6,31)}=\right.$ $17.51, p<0.01]$ of the variability in the infection rates for NHB population. The final model for the NHW infection rates consists of obesity and poverty as the only explanatory variables. This model accounts for $66 \%\left[R^{2}=0.66, R_{a d j}^{2}=0.64, F_{(2,37)}=\right.$ $37.89, p<0.01]$ of the variability in the NHB infection rates. Note that the final NHB infection model uses 40 states instead of 38 states in the preliminary model. This discrepancy is because of the unavailability of data for the NHB community for all the explanatory variables, as discussed in section 2.4.3. The preliminary model with $\mathrm{NHB}$ mortality rates as the response variable accounts for $77 \%\left[R^{2}=0.77, R_{a d j}^{2}=0.73, F_{(6,31)}=\right.$ $17.51, p<0.01]$ of the variability in the NHB mortality rates. The final model for the NHB mortality also consists of only obesity 
TABLE 7 | Linear models with infection and mortality rates as response variables for NHB.

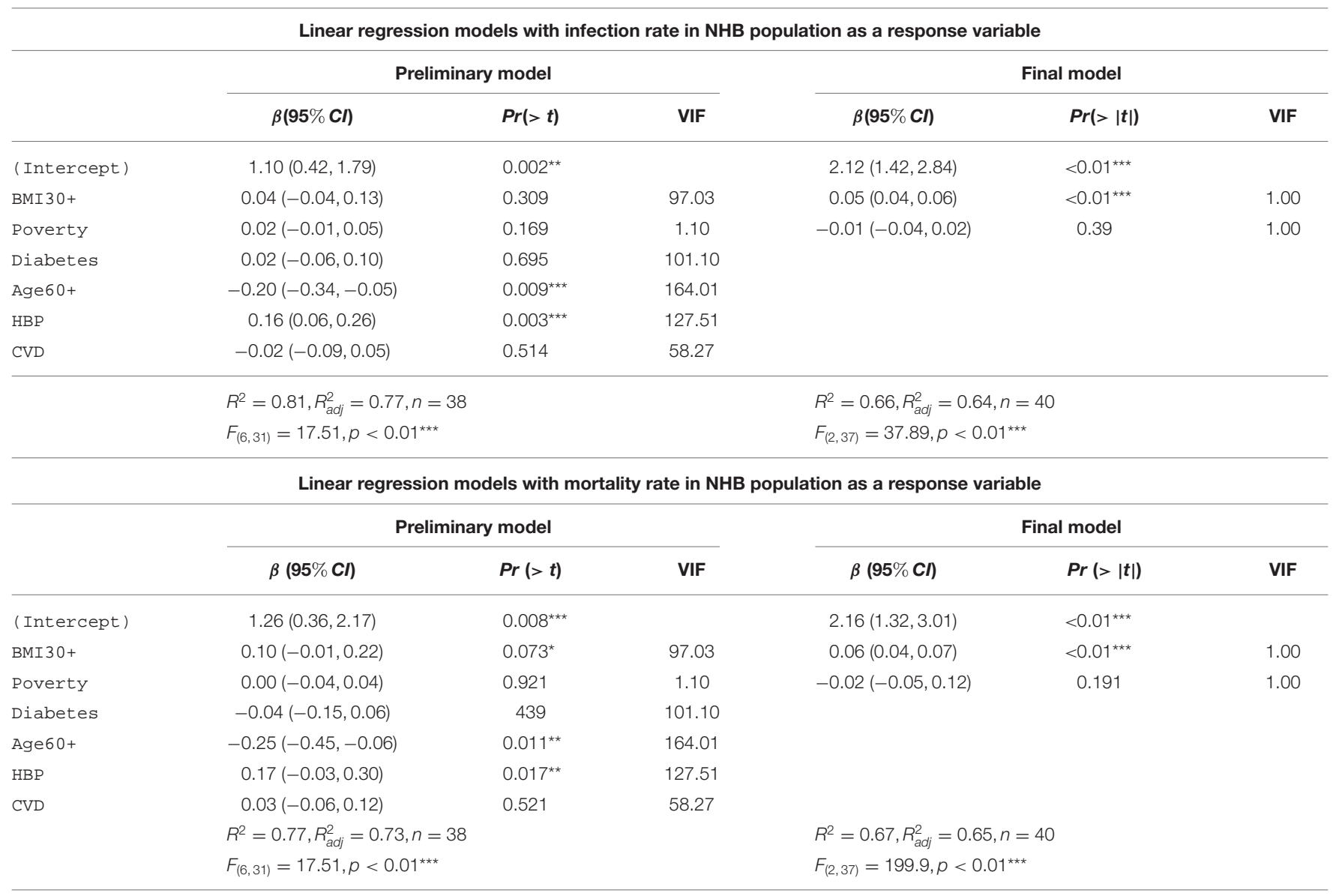

The first column shows preliminary model along with the VIFs for each explanatory variable. The second column indicates the model obtained using maximum VIF elimination algorithm.

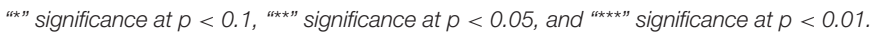

and poverty as the explanatory variables. This NHB mortality final model accounts for $67 \%\left[R^{2}=0.67, R_{a d j}^{2}=0.65, F_{(2,37)}=\right.$ $199.9, p<0.01]$ of the variability in the NHB mortality rates.

The Table 8 depicts the linear models with infection rate and mortality rate as response variables for the Hispanic group. The preliminary model for infection rates in the Hispanic community accounts for $67 \%\left[R^{2}=0.67, R_{a d j}^{2}=0.60, F_{(6,26)}=8.97, p<\right.$ $0.01]$ of the variability in the infection rates for the Hispanic population. The final model for the Hispanic infection rates consists of diabetes and poverty as the explanatory variables. This model accounts for $51 \%\left[R^{2}=0.51, R_{a d j}^{2}=0.48, F_{(6,25)}=\right.$ $9.98, p<0.01]$ of the variability in the Hispanic infection rates. The preliminary model with mortality rates among the Hispanic community as the response variable accounts for $71 \%$ $\left[R^{2}=0.71, R_{a d j}^{2}=0.63, F_{(6,25)}=9.98, p<0.01\right]$ of the variability in the Hispanic mortality rates. The final model for Hispanic mortality consists of advanced age and poverty as the explanatory variables. Note that advanced age is the most significant explanatory variable in the final mortality model in the Hispanic group, whereas having diabetes was the most significant variable predicting infection in the Hispanic community. This final model accounts for $55 \%\left[R^{2}=0.55, R_{a d j}^{2}=0.53, F_{(2,39)}=\right.$ $23.93, p<0.01]$ of the variability in the Hispanic mortality rates. We note that the final model for the Hispanic mortality includes 42 states, whereas the preliminary model has only 32 states due to lack of data availability.

Adjusted $R^{2}$ value for the regression model for NHW mortality was much higher (0.77) in comparison to NHB (0.65) and Hispanic (0.53). However, all six models showed statistical significance and satisfied normality tests for the residual values. Indeed, the Lilliefors normality test applied to the residuals obtained from each of these models revealed that the residuals were normally distributed with $p>0.001$. The histograms, and the QQ plots for the residuals are provided in Figure 3.

\subsection{Results From the Geographically Weighted Regression}

The geographically weighted regression yields coefficients for each risk factor for every state. We show the state-wise coefficients for the most significant explanatory variable for each ethnicity in Figure 4. Empty spaces for states in Figure 4 indicate that ethnicity-wise data was not available for those states for the 
TABLE 8 | Linear models with infection and mortality rates as response variables for Hispanic.

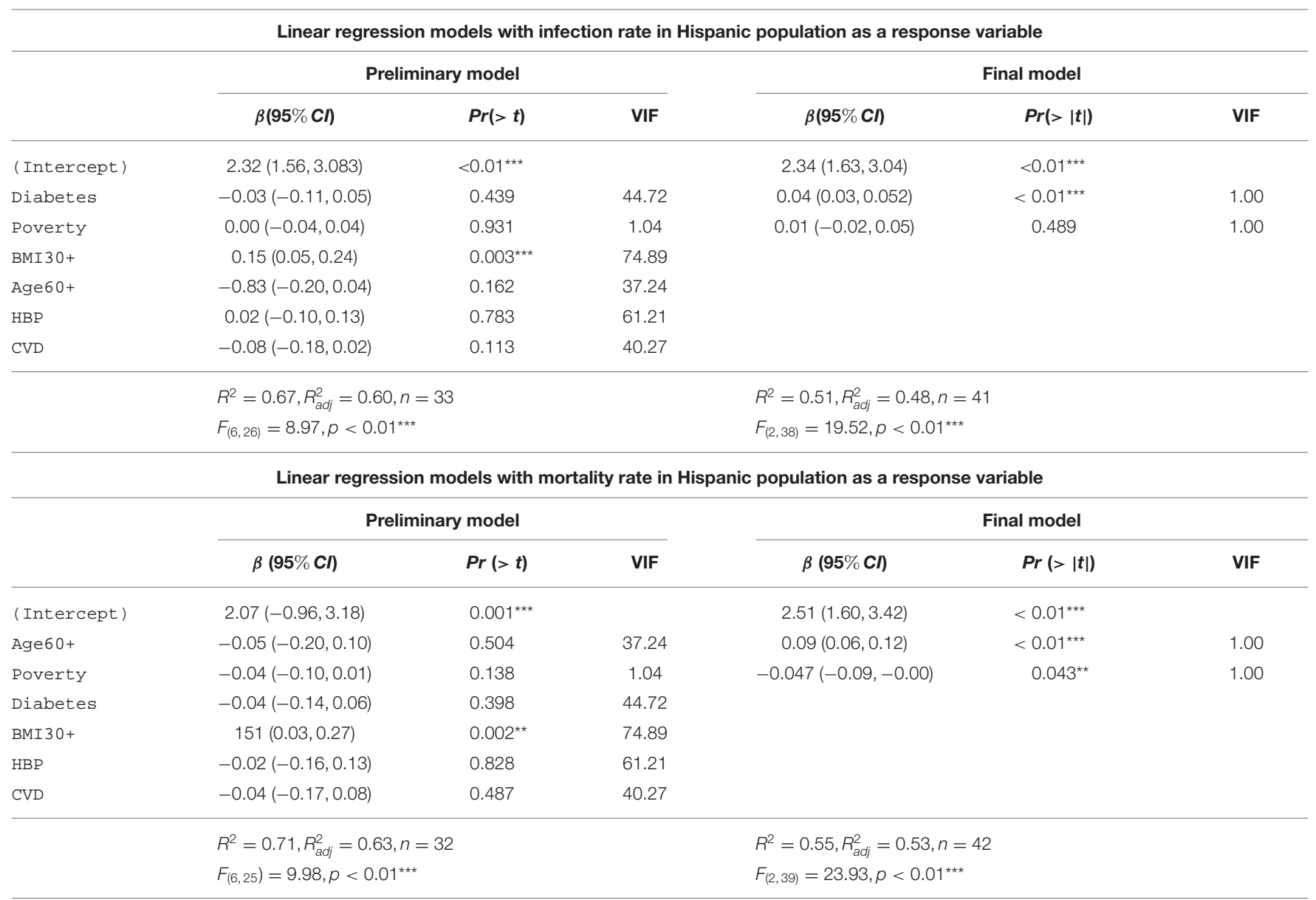

The first column shows preliminary model along with the VIFs for each explanatory variable. The second column indicates the model obtained using maximum VIF elimination algorithm.

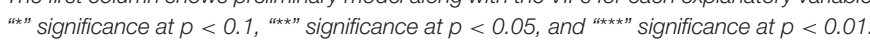

corresponding risk factors. The $p$-values for the most significant explanatory variable and state-wise $R^{2}$ values obtained from the GWR model are included in Supplementary Figures S4, S5.

\section{DISCUSSION}

Our analysis of the nationwide data revealed that geographical location, and other COVID-19 risk factors affect different ethnicities in a dissimilar way. We observed that the disparate burden of the pandemic was most prominent on the NHB and Hispanic communities. This observation is supported by Anyane-Yeboa et al. (56) and Escoba et al. (57) other studies. In particular, the rate of infection was exceptionally high for the Hispanic community compared to their population share. Discordant impact on NHB and Hispanic populations has been reported by Centers for Disease Control (58) and studied using data from metropolitan cities and combining selected states, but the nationwide study is limited. In our work, this effect was observed in the four US regions separately and also when all the states' data was aggregated. When considered the four regions individually, we found that the excessive infection rate in the Hispanic community was most prominent in the South region. However, compared to the Hispanic group's infection rates, their mortality rates were statistically lower in all regions of the US. This apparent discrepancy could be because the Hispanic community is the youngest of the four ethnic groups considered in our study (59). The infection rate of NHB population was higher compared to their population share in the Midwest, and the Northeast than other regions.

The correlation analysis confirmed that the COVID-19 related risk factors such as advanced age, cardiovascular disease, diabetes, hypertension, and obesity are highly interrelated. This finding is consistent with numerous studies. For example, Mokdad et al. (60) show that obesity (BMI $\geq 30$ ) was significantly associated with diabetes, hypertension, high cholesterol, asthma, and arthritis. Wilson and Kannel (61) conclude that obesity and diabetes are associated with atherogenic risk factors. Abdullah et al. (62) also conclude that obesity is associated with type 2 diabetes. We also found that "within" an ethnic group, poverty was uncorrelated or weakly correlated with infections and mortality for all three ethnic groups, implying that poverty 

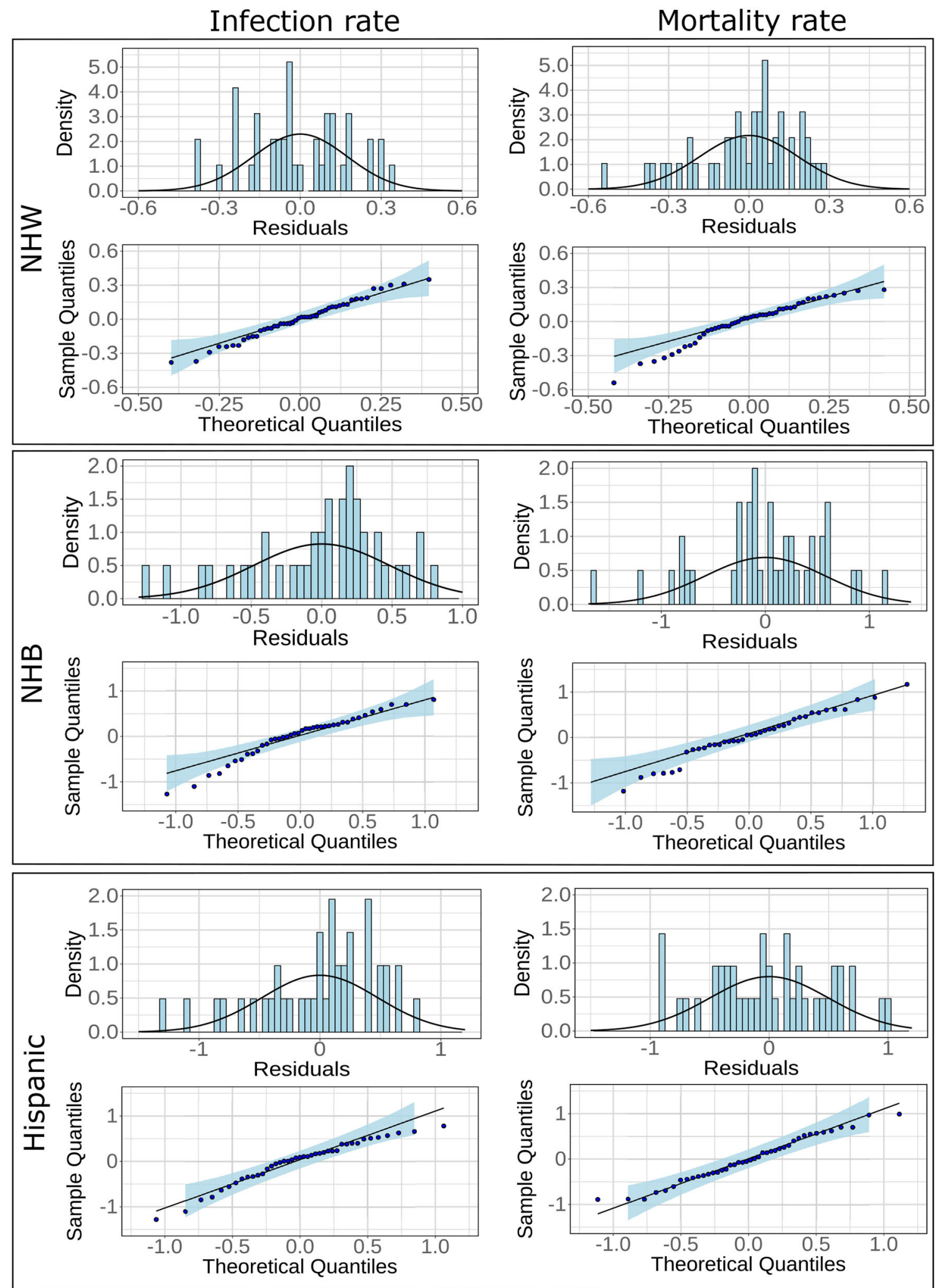

FIGURE 3 | Histograms and fitted normal curves along with QQ plots of the residuals for linear regression for NHW, NHB, and Hispanics groups. The linear models are based on infections and mortality rates as response variables. 


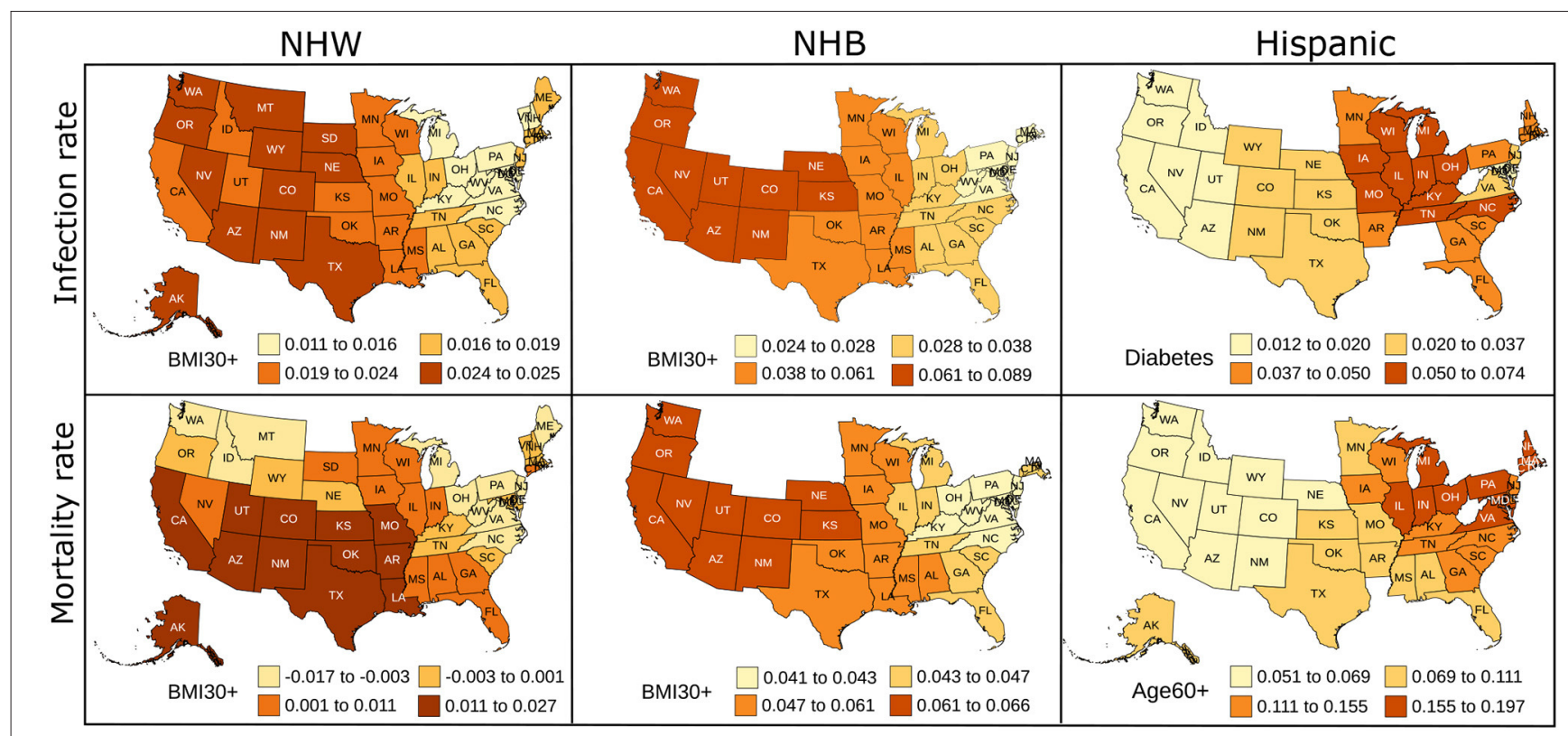

FIGURE 4 | Map showing the coefficient values for the most significant variables for the GWR models constructed on predictors used in linear regression for NHW, $\mathrm{NHB}$, and Hispanics data. The states with no color indicate that the ethnicity-wise data on the corresponding risk factors was not available in those states.

is an "independent" risk factor for COVID-19. This finding is supported by Elgar et al. (32) and Oronce et al. (33) which we discussed in section 1.4.

After eliminating variables with high multicollinearity, we formulated robust and parsimonious linear models for NHW, $\mathrm{NHB}$, and Hispanic populations. The linear models described in section 3.3 reveal that "obesity" encapsulates many other codependent risk factors for the infection and mortality in NHW and NHB groups. This finding is expected in light of numerous studies $(61,63)$. Obesity and diabetes are well-established risk factors for COVID-19. In these two conditions, adipose tissue is compromised, which can directly or indirectly get involved in interaction with SARS-CoV-2, the pathogen responsible for COVID-19 disease (64). Thus, it is not surprising that obesity highly influences the regression models for NHW and NHB with death rate as a response variable. However, the degree of influence of obesity on infection rates and mortality is noteworthy, with obesity emerging as the most significant factor contributing to the infection rates and mortality for the NHW and NHB groups.

The Hispanic community markedly differs from NHW, and NHB with respect to the results of the linear models. Diabetes was the most significant factor for infection rate in Hispanics, while advanced age emerged as most significant for mortality. The effect of advanced age on Hispanic mortality could be also due to the relatively younger, and thus working-age, population of Hispanics (59) in the US.

The regression models indicate a strong association of poverty with a high infection rate, followed by death for all ethnic groups studied. This finding is in agreement with several studies focusing on the association of low socioeconomic status, which increases the exposure to COVID-19 $(31,65)$. People with low socioeconomic status avail healthcare services at an advanced stage of illness, thus experience a worse prognosis. The disease burden associated with obesity is linked to socioeconomic status and race (28). Ethnic minorities and populations with low socioeconomic status have been disproportionately affected in previous pandemics $(5,6,8)$. Evidence from the COVID-19 pandemic is not an exception to the above fact. To this end, public health strategies to control the current and future pandemics need to take these ethnicity-specific effects into account to mitigate the spread and severity of the disease.

The linear regression furnishes global and static coefficients for the explanatory variables. However, the geographically weighted regression gives coefficients that are geographically varying. We see in Figure 4 the variability in the coefficients of the GWR. We note that the neighboring states seem to have similar coefficients, indicating similarity in the risk factors in nearby states. Obesity is the most prominent risk factor amongst the NHW and NHB populations, and diabetes and advanced age seem to be more influential in the Hispanic community. The GWR results for the Hispanic group show more variability than the NHW group, which could be due to the higher percentage of people of Hispanic origin in southern and western states. The local $R^{2}$ map in Supplementary Figure S5 also indicates that the GWR model fits the NHW and NHB groups better than the Hispanic group. We plan to explore the geographical variation of the risk factors in more detail in future work.

\subsection{Limitations}

As discussed in section 2.2 and noted by other researchers $(36,38)$ there is a lack of consistency and availability of COVID-19 related data. Our study does not include data from the state of New York, since the state does not make the ethnicity wise data 
available. Moreover, the data we use is state wise statistics of the various risk factors. However, we note that the observations made using such data is consistent with those made by other researchers.

\subsection{Practical Implications of the Study}

Although racial and ethnic disparities in COVID-19 infections and mortality are becoming increasingly clear from several studies based on available data, drivers of these disparate outcomes remain less understood at a national level. Our models, based on the nationwide data, indicate that "obesity" effectively encapsulates the effect of other co-dependent factors for NHW, and NHB populations (section 3.3). The link between COVID19 infection severity and obesity is noted by Watanabe et al. (66) even in the early stages of the pandemic. Similarly, during the H1N1 pandemic of $2009(67,68)$ observed that obesity was associated with higher mortality.

Another implication from our work is that the Hispanic community is more susceptible to the COVID-19 infection. This observation is valid throughout the US. This situation could be remedied via public policy changes and awareness of the issue. The disproportionate impact of COVID-19 on the minority population is largely attributed to existing socioeconomic inequities. The low-income minority population are often compelled to work in an environment with higher risk of disease exposure, live in a crowded accommodation, and lack adequate access to healthcare. The government support to lowincome families in the form of the CARES Act, Consolidated Appropriations Act, 2021, Department of Treasury US (69) and the American Rescue Plan Act of 2021 (70) are critical but might not be sufficient to fully mitigate observed disparity in infection and mortality rates. Our analysis indicates that certain subpopulations of the minority population are at higher risk of COVID-19 infection and mortality. Identifying these vulnerable subpopulations, such as Hispanics with diabetes or age over 60 years, and prioritizing additional attention to these populations could enable a more efficient allocation and utilization of resources. Increased effort toward educating and raising awareness on COVID-19 and associated risk factors could also be an effective method to develop community resilience. One potential avenue to improve awareness on COVID-19 will be through recruiting volunteers to educate the vulnerable population. For example, "Philly counts" (71), a program supported by the Philadelphia Department of Public Health, initially created for Census 2020, currently helps direct community engagement efforts for the COVID-19 vaccine. Extending similar initiatives to populations with major risk factors such as obesity could result in a major beneficial impact on overall COVID-19 burden.

\section{CONCLUSION}

Several researchers have concluded that several health conditions, poverty, and geographical location affect the COVID-19 prognosis. Studies have shown that the COVID-19 pandemic has impacted some minorities in the US more severely than other groups. Our work focused on quantifying this distinct effect of various COVID-19 risk factors on different ethnicities in the US during the first pandemic wave.

To this effect, we included Non-Hispanic White, NonHispanic Black, Hispanic, Non-Hispanic Asians. Our work has revealed differences in the way the COVID-19 pandemic affected various ethnic groups. We observed that the infection rates in the Hispanic population were disproportionately larger than the share of their population across all regions of the US. This effect was most prominent in the South region. The NHA populations consistently had lower infection rates and mortality rates compared to their population. Furthermore, we studied the following risk factors in this work: advanced age, obesity, cardiovascular diseases, diabetes, hypertension, and poverty for NHW, NHB, and Hispanic populations. We aimed to quantify the different effects of these risk factors on various ethnicities. To this end, we constructed linear models with infection and mortality rates as the response variables. We eliminated variables causing multicollinearity from our models, leading to robust linear models. Our models indicate that "obesity" parsimoniously describes the impact of other co-dependent comorbidities for NHW and NHB populations (section 3.3). However, for the infection rates in the Hispanic group, the factor leading to the robust linear model was the prevalence of diabetes. On the other hand, advanced age was more significant for COVID19 related mortality for the Hispanic community. We also established "poverty" as an independent risk factor for infection and mortality amongst the three ethnicities: NHW, NHB, and Hispanics. The findings in this study quantified ethnicityspecific effects of COVID-19 risk factors, which we hope could be mollified with public policy interventions and community engagement.

\section{DATA AVAILABILITY STATEMENT}

Publicly available datasets were analyzed in this study. This data can be found here: https://github.com/prashantva/Covid19-Ethnicity.

\section{AUTHOR CONTRIBUTIONS}

PA: writing-review, conceptualization, editing, investigation, and analysis. JC: data collection. VK: data curation, formal analysis, visualization, and coding. SM: writing-original draft, methodology, formal analysis, and project administration. SS: supervision, conceptualization, validation, and editing. All authors contributed to the article and approved the submitted version.

\section{SUPPLEMENTARY MATERIAL}

The Supplementary Material for this article can be found online at: https://www.frontiersin.org/articles/10.3389/fpubh. 2021.743003/full\#supplementary-material 


\section{REFERENCES}

1. Chartier K, Caetano R. Ethnicity and health disparities in alcohol research. Alcohol Res. (2010) 33:152.

2. Barr DA. Health Disparities in the United States: Social Class, Race, Ethnicity, and Health. Baltimore, MD: JHU Press (2014).

3. Dressler WW, Oths KS, Gravlee CC. Race and ethnicity in public health research: models to explain health disparities. Annu Rev Anthropol. (2005) 34:231-52. doi: 10.1146/annurev.anthro.34.081804.120505

4. Braun L. Race, ethnicity, and health: can genetics explain disparities? Perspect Biol Med. (2002) 45:159-74. doi: 10.1353/pbm.2002.0023

5. Khunti K, Platt L, Routen A, Abbasi K. Covid-19 and ethnic minorities: an urgent agenda for overdue action. Br Med J Publish Group. (2020) doi: 10.1136/bmj.m2503

6. Kabarriti R, Brodin NP, Maron MI, Guha C, Kalnicki S, Garg $\mathrm{MK}$, et al. Association of race and ethnicity with comorbidities and survival among patients with COVID-19 at an urban medical center in New York. JAMA Netw Open. (2020) 3:e2019795-e2019795. doi: 10.1001/jamanetworkopen.2020.19795

7. Owen WF, Carmona R, Pomeroy C. Failing another national stress test on health disparities. JAMA. (2020) 323:1905-6. doi: 10.1001/jama.2020.6547

8. Dobin D, Dobin A. Racial/ethnic and socioeconomic disparities of Covid19 attacks rates in Suffolk County communities. arXiv[Preprint]. (2020). arXiv:2004.12175.

9. Moore JT, Ricaldi JN, Rose CE, Fuld J, Parise M, Kang GJ, et al. Disparities in incidence of COVID-19 among underrepresented racial/ethnic groups in counties identified as hotspots during June 5-18, 2020-22 states, February-June (2020). Morbid Mortal Wkly Rep. (2020) 69:1122. doi: 10.15585/mmwr.mm6933e1

10. Adhikari S, Pantaleo NP, Feldman JM, Ogedegbe O, Thorpe L, Troxel AB. Assessment of community-level disparities in coronavirus disease 2019 (COVID-19) infections and deaths in large US metropolitan areas. JAMA Netw Open. (2020) 3:e2016938. doi: 10.1001/jamanetworkopen.2020. 16938

11. Sun Z, Zhang H, Yang Y, Wan H, Wang Y. Impacts of geographic factors and population density on the COVID-19 spreading under the lockdown policies of China. Scie Total Environ. (2020) 746:141347. doi: 10.1016/j.scitotenv.2020.141347

12. Breen R, Ermisch J. The distributional impact of COVID-19: Geographic variation in mortality in England. Demogr Res. (2021) 44:397-414. doi: 10.4054/DemRes.2021.44.17

13. Desmet K, Wacziarg R. JUE Insight: Understanding spatial variation in COVID-19 across the United States. J Urban Econ. (2021) 103332. doi: $10.1016 /$ j.jue.2021.103332

14. Stephens-Davidowitz S. "The cost of racial animus on a black candidate: evidence using Google search data”. J Public Econ. (2014). 118:26-40. doi: 10.1016/j.jpubeco.2014.04.010

15. Drucker DJ. Coronavirus infections and type 2 diabetes-shared pathways with therapeutic implications. Endocr Rev. (2020) 41:457-70. doi: 10.1210/endrev/bnaa011

16. Muniyappa R, Gubbi S. COVID-19 pandemic, coronaviruses, and diabetes mellitus. Am J Physiol Endocrinol Metab. (2020) 318:E736-41. doi: 10.1152/ajpendo.00124.2020

17. Orioli L, Hermans MP, Thissen JP, Maiter D, Vandeleene B, Yombi JC. COVID-19 in diabetic patients: related risks and specifics of management. In: Annales d'endocrinologie. Vol. 81. Elsevier (2020). p. 101-9. doi: 10.1016/j.ando.2020.05.001

18. Yan Y, Yang Y, Wang F, Ren H, Zhang S, Shi X, et al. Clinical characteristics and outcomes of patients with severe covid-19 with diabetes. BMJ Open Diabetes Res Care. (2020) 8:e01343. doi: 10.1136/bmjdrc-2020001343

19. Richardson S, Hirsch JS, Narasimhan M, Crawford JM, McGinn T, Davidson KW, et al. Presenting characteristics, comorbidities, and outcomes among 5700 patients hospitalized with COVID-19 in the New York City area. JAMA. (2020) 323:2052-9. doi: 10.1001/jama.2020.6775

20. Sun C, Zhang X, Dai Y, Xu X, Zhao J. Clinical analysis of 150 cases of 2019 novel coronavirus infection in Nanyang City, Henan Province. Zhonghua jie he he hu xi za zhi= Zhonghua Jiehe he
Huxi Zazhi= Chin J Tuberculosis Respiratory Dis. (2020) 43:E042. doi: 10.3760/cma.j.cn112147-20200224-00168

21. Gao F, Zheng KI, Wang XB, Sun QF, Pan KH, Wang TY, et al. Obesity is a risk factor for greater COVID-19 severity. Diabetes Care. (2020) 43:e72-4. doi: $10.2337 / \mathrm{dc} 20-0682$

22. Busetto L, Bettini S, Fabris R, Serra R, Dal Pra C, Maffei P, et al. "Obesity and COVID-19: an Italian snapshot". Obesity. (2020) 28:1600-5. doi: $10.1002 /$ oby. 22918

23. Sharp JT, Henry JP, Sweany SK, Meadows WR, Pietras RJ. The total work of breathing in normal and obese men. J Clin Invest. (1964) 43:728-39. doi: 10.1172/JCI104957

24. Li AM, Chan D, Wong E, Yin J, Nelson EAS, Fok TF. The effects of obesity on pulmonary function. Arch Dis Child. (2003) 88:361-3. doi: $10.1136 /$ adc. 88.4 .361

25. Anderson RM, Fraser C, Ghani AC, Donnelly CA, Riley S, Ferguson NM, et al. Epidemiology, transmission dynamics and control of SARS: the 2002\&\#x2013;2003 epidemic. Philos Trans R Soc Lond B Biol Sci. (2004) 359:1091-105. doi: 10.1098/rstb.2004.1490

26. Ma C, Gu J, Hou P, Zhang L, Bai Y, Guo Z, et al. "Incidence, clinical characteristics and prognostic factor of patients with COVID-19: a systematic review and meta-analysis". medRxiv. (2020) doi: 10.1101/2020.03.17.2003 7572

27. CDC dataset. Provisional COVID-19 Deaths by Sex and Age. (2020). Available online at: https://data.cdc.gov/NCHS/Provisional-COVID-19-Deaths-bySex-and-Age/9bhg-hcku

28. Paeratakul S, Lovejoy JC, Ryan DH, Bray GA. The relation of gender, race and socioeconomic status to obesity and obesity comorbidities in a sample of US adults. Int J Obes. (2002) 26:1205-10. doi: 10.1038/sj.ijo.080 2026

29. Sturm R, Hattori A. Morbid obesity rates continue to rise rapidly in the United States. Int J Obes. (2013) 37:889-91. doi: 10.1038/ijo.2012.159

30. Kuzawa CW, Sweet E. Epigenetics and the embodiment of race: developmental origins of US racial disparities in cardiovascular health. Am J Hum Biol. (2009) 21:2-15. doi: 10.1002/ajhb.20822

31. Patel J, Nielsen F, Badiani A, Assi S, Unadkat V, Patel B, et al. Poverty, inequality and COVID-19: the forgotten vulnerable. Public Health. (2020) 183:110. doi: 10.1016/j.puhe.2020.05.006

32. Elgar FJ, Stefaniak A, Wohl MJA. The trouble with trust: time-series analysis of social capital, income inequality, and COVID-19 deaths in 84 countries. Soc Sci Med. (2020) 263:113365. doi: 10.1016/j.socscimed.2020. 113365

33. Oronce CIA, Scannell CA, Kawachi I, Tsugawa Y. Association between statelevel income inequality and COVID-19 cases and mortality in the USA. J Gen Intern Med. (2020) 35:2791-3. doi: 10.1007/s11606-020-05971-3

34. Hsu CC, Lee CH, Wahlqvist ML, Huang HL, Chang HY, Chen L, et al. Poverty increases type 2 diabetes incidence and inequality of care despite universal health coverage. Diabetes Care. (2012) 35:2286-92. doi: 10.2337/dc11-2052

35. Census Bureau US. 2010 Census Regions and Divisions of the United States. (2018). Available online at: https://www2.census.gov/geo/pdfs/maps-data/ maps/reference/us_regdiv.pdf

36. Carroll SR, Akee R, Chung P, Cormack D, Kukutai T, Lovett R, et al. Indigenous peoples' data during COVID-19: from external to internal. Front Sociol. (2021) 6:62. doi: 10.3389/fsoc.2021.617895

37. Hatcher SM, Agnew-Brune C, Anderson M, Zambrano LD, Rose CE, Jim MA, et al. COVID-19 among American Indian and Alaska native persons23 states, January 31-July 3, (2020). Morbid Mortal Wkly Rep. (2020) 69:1166. doi: 10.15585/mmwr.mm6934e1

38. Talagadadeevi T, Iyer S, Saurer D. An analysis of limitations for demographic data reporting on State COVID-19 dashboards. (2020). Available online at: https://covid19dataproject.org/wp-content/uploads/2020/ 09/9_27-vertical-demographic-poster_good.pdf

39. KFF Race data. Population Distribution by Race/Ethnicity. (2020). Available online at: https://www.kff.org/other/state-indicator/distribution-byraceethnicity/

40. KFFCovid-19 data. Population Distribution by Race/Ethnicity. (2020) Available online at: https://github.com/KFFData/COVID-19-Data

41. ACL dataset. Administration for Community Living. (2018). Available online at https://p-agid-2021-wapp1.azurewebsites.net/StateProfiles/ 
42. AHR Diabetes data. Public Health Impact: Diabetes. (2019). Available online at: https://www.americashealthrankings.org/explore/annual/measure/ Diabetes/state/ALL? edition-year $=2019$

43. National Institute of Health NH, Lung, Institute B, of Diabetes NI, Digestive, (US) KD. Clinical Guidelines on the Identification, Evaluation, and Treatment of Overweight and Obesity in Adults-The Evidence Report. Vol. 6. Bethesda, MD: National Heart, Lung, and Blood Institute. (1998).

44. AHR Obesity data. Public Health Impact: Obesity. (2020). Available online at: https://www.americashealthrankings.org/explore/annual/measure/ Obesity/state/ALL

45. AHR CVD data. Public Health Impact: Cardiovascular Diseases. (2019). Available online at: https://www.americashealthrankings.org/explore/annual/ measure/Obesity/state/ALL

46. AHR HBP data. Public Health Impact: High Blood Pressure. (2019). Available online at: https://www.americashealthrankings.org/explore/annual/measure/ Hypertension/state/ALL

47. KFF Poverty data. Poverty Rate by Race/Ethnicity. (2019). Available online at: https://www.kff.org/other/state-indicator/poverty-rate-by-raceethnicity

48. Kruskal WH, Wallis WA. Use of ranks in one-criterion variance analysis. J Am Stat Assoc. (1952) 47:583-21. doi: 10.1080/01621459.1952.10483441

49. Dunn OJ. Multiple comparisons among means. J Am Stat Assoc. (1961) 56:52-64. doi: 10.1080/01621459.1961.10482090

50. Bland JM, Altman DG. Statistics notes. Logarithms. BMJ. (1996) 312:700. doi: 10.1136/bmj.312.7032.700

51. Belsley DA, Kuh E, Welsch RE. Regression Diagnostics: Identifying Influential Data and Sources of Collinearity. Hoboken, NJ: John Wiley \& Sons;. (2004).

52. Neter J, Kutner MH, Nachtsheim CJ, Wasserman W. Applied Linear Statistical Models. 4th ed. Chicago, IL: IRWIN (1996).

53. Kutner MH, Nachtsheim CJ, Neter J. Applied Linear Regression Models. New York, NY: McGraw Hill (2004).

54. Lilliefors HW. On the Kolmogorov-Smirnov test for normality with mean and variance unknown. J Am Stat Assoc. (1967) 62:399-402. doi: 10.1080/01621459.1967.10482916

55. Brunsdon C, Fotheringham S, Charlton M. Geographically weighted regression. J R Stat Soc D. (1998) 47:431-43. doi: 10.1111/1467-9884. 00145

56. Anyane-Yeboa A, Sato T, Sakuraba A. Racial disparities in COVID-19 deaths reveal harsh truths about structural inequality in America. J Intern Med. (2020) 288:479-80. doi: 10.1111/joim.13117

57. Escobar GJ, Adams AS, Liu VX, Soltesz L, Chen YFI, Parodi SM, et al. Racial disparities in COVID-19 testing and outcomes: retrospective cohort study in an integrated health system. Ann Intern Med. (2021) 174:786-93. doi: 10.7326/M20-6979

58. for Disease Control C, Prevention, et al. COVID-19 hospitalization and death by race/ethnicity. (2020). Available online at: https://www.cdc.gov/ coronavirus/2019-ncov/covid-data/investigations-discovery/hospitalizationdeath-by-race-ethnicity.html (accessed September 9, 2021).

59. Patten E. The Nation's Latino Population Is Defined by Its Youth. (2016). Available online at: https://www.pewresearch.org/hispanic/2016/04/20/thenations-latino-population-is-defined-by-its-youth/

60. Mokdad AH, Ford ES, Bowman BA, Dietz WH, Vinicor F, Bales VS, et al. Prevalence of obesity, diabetes, and obesity-related health risk factors, 2001. JAMA. (2003) 289:76-9. doi: 10.1001/jama.289.1.76
61. Wilson PW, Kannel WB. Obesity, diabetes, and risk of cardiovascular disease in the elderly. Am J Geriatr Cardiol. (2002) 11:119-24. doi: 10.1111/j.1076-7460.2002.00998.x

62. Abdullah A, Peeters A, de Courten M, Stoelwinder J. The magnitude of association between overweight and obesity and the risk of diabetes: a meta-analysis of prospective cohort studies. Diabetes Res Clin Pract. (2010) 89:309-19. doi: 10.1016/j.diabres.2010.04.012

63. Dietz W, Santos-Burgoa C. Obesity and its implications for COVID-19 mortality. Obesity. (2020) 28:1005-5. doi: 10.1002/oby.22818

64. Kruglikov IL, Shah M, Scherer PE. Obesity and diabetes as comorbidities for COVID-19: underlying mechanisms and the role of viral-bacterial interactions. Elife. (2020) 9:e61330. doi: 10.7554/eLife.61330

65. Yechezkel M, Weiss A, Rejwan I, Shahmoon E, Ben-Gal S, Yamin D. Human mobility and poverty as key drivers of COVID-19 transmission and control. BMC Public Health. (2021) 21:1-13. doi: 10.1186/s12889-021-10561-x

66. Watanabe M, Risi R, Tuccinardi D, Baquero CJ, Manfrini S, Gnessi L. Obesity and SARS-CoV-2: a population to safeguard. Diabetes Metab Res Rev. (2020) 36:e3325. doi: 10.1002/dmrr.3325

67. Morgan OW, Bramley A, Fowlkes A, Freedman DS, Taylor TH, Gargiullo $\mathrm{P}$, et al. Morbid obesity as a risk factor for hospitalization and death due to 2009 pandemic influenza A (H1N1) disease. PLoS ONE. (2010) 5:e9694. doi: 10.1371/journal.pone.0009694

68. Louie JK, Acosta M, Samuel MC, Schechter R, Vugia DJ, Harriman K, et al. A novel risk factor for a novel virus: obesity and 2009. Pandemic Influenza A (H1N1). Clin Infect Dis. (2011) 52:301-12. doi: 10.1093/cid/ciq152

69. Department of Treasury US. About the CARES Act and the Consolidated Appropriations Act. (2020). Available online at: https://home.treasury.gov/ policy-issues/coronavirus/about-the-cares-act

70. Department of Treasury US. FACT SHEET: The American Rescue Plan Will Deliver Immediate Economic Relief to Families. (2021). Available online at: https://home.treasury.gov/policy-issues/coronavirus/about- the-cares-act

71. City of Philladelphia. Philly Counts: Empowering Residents to Take Action and be Agents of Change in Their Communities. (2021). Available online at: https:// www.phila.gov/programs/philly-counts-2020/

Conflict of Interest: The authors declare that the research was conducted in the absence of any commercial or financial relationships that could be construed as a potential conflict of interest.

Publisher's Note: All claims expressed in this article are solely those of the authors and do not necessarily represent those of their affiliated organizations, or those of the publisher, the editors and the reviewers. Any product that may be evaluated in this article, or claim that may be made by its manufacturer, is not guaranteed or endorsed by the publisher.

Copyright (C) 2021 Athavale, Kumar, Clark, Mondal and Sur. This is an open-access article distributed under the terms of the Creative Commons Attribution License (CC BY). The use, distribution or reproduction in other forums is permitted, provided the original author(s) and the copyright owner(s) are credited and that the original publication in this journal is cited, in accordance with accepted academic practice. No use, distribution or reproduction is permitted which does not comply with these terms. 